\title{
7-Nitro-5,10,15,20-tetraphenylporphyrin Dichloromethane Solvate
}

\section{O. Senge}

\begin{abstract}
The title compound shows significant conformational distortion in the vicinity of the $\beta$-nitro group. This is attributed to steric interaction between a meso-phenyl and the $\beta$-nitro group. The overall degree of conformation distortion is moderate, the average deviation of the 24 macrocycle atoms from their least- squares plane being $0.08 \AA$. The largest displacements ( $0.33 \AA$ from the plane of the four pyrrole nitrogen atoms) were found for the $\mathrm{C}_{\mathrm{b}}$-positions of the nitrated pyrrole ring.
\end{abstract}

\section{Comment}

The title compound was investigated with regard to potential steric interactions between the $\beta$-nitro group and the neighboring meso-phenyl substituent. The overall degree of macrocycle distortion is moderate, the average deviation of the 24 macrocycle atoms from their least-square plane being only $0.088 \AA$. Nevertheless, a more localized conformational influence of the nitro-group is observed. The average displacement of the $\mathrm{C}_{\mathrm{b}}$-carbons from the least-square plane of the four pyrrole nitrogen atoms ( $4 \mathrm{~N}$-plane) is $0.17 \AA$. The respective average displacement values for the $\mathrm{C}_{\mathrm{b}}$ carbons of individual pyrrole rings are: ring I (with nitro group) $0.33 \AA$, ring II $0.25 \AA$, ring III $0.03 \AA$, and ring IV $0.07 \AA$. The plane of the pyrrole rings are tilted against the $4 \mathrm{~N}$-plane by $9^{\circ}$ (ring I), $7.1^{\circ}$ (ring II), $0.9^{\circ}$ (ring III), and $3.0^{\circ}$ (ring IV). Thus, the macrocycle shows definite distortions in the vicinity of the meso- phenyl/nitro-interaction which become progressively smaller which increasing distance from the $\beta$-nitro group. Displacements of the meso-carbons from the $4 \mathrm{~N}$-plane are in the order of $0.02-0.04 \AA$. The nitro group is tilted against the $4 \mathrm{~N}$-plane by $48.4^{\circ}$. There is no evidence for increased $\pi$ - $\pi$-overlap between the nitro-group and the aromatic macrocycle.

The molecules form well separated layers with no evidence of $\pi$ - stacking. The closest intermolecular contacts observed were $\mathrm{N} 21-\mathrm{Cl} 2 \mathrm{~S}(3.15 \AA)$ and $\mathrm{O} 1-\mathrm{H} 202(2.51 \AA)$. Related structures are a planar high-spin Ni(II) complex of 2,3,7,8,12,13,17-heptanitro-5,10,15,20- tetrakis(2,6-dichlorophenyl)porphyrin (Ozette et al., 1997) and a saddle-distorted 7,12-dinitro-5,10,15,20-tetraphenylporphyrin (Dahal et al., 1994). $\beta$-Nitroporphyrins have recently been shown to be excellent precursors for the preparation of fused pyrrolo- porphyrins (Jaquinod et al., 1996).

\section{Experimental}

The compound was prepared synthetically as described by Giraudeau, A. et al. (1979). Crystals were grown from $\mathrm{CH}_{2} \mathrm{Cl}_{2} /$ n-hexane and mounted according to Hope (1994).

\section{Computing details}

Data collection: Siemens P3; cell refinement: Siemens P3; data reduction: XDISK, SHELXTL-Plus (Sheldrick, 1994); program(s) used to solve structure: SHELXS86 (Sheldrick, 1990); program(s) used to refine structure: SHELXL93 (Sheldrick, 
1993); molecular graphics: XP, SHELXTL-Plus (Sheldrick, 1994); software used to prepare material for publication: SHELXL, SHELXTL-Plus (Sheldrick, 1994).

\section{7-Nitro-5,10,15,20-tetraphenylporphyrin}

\section{Crystal data}

$\mathrm{C}_{44} \mathrm{H}_{29} \mathrm{~N}_{5} \mathrm{O}_{2} \cdot \mathrm{C}_{1} \mathrm{H}_{2} \mathrm{Cl}_{2}$

$M_{r}=744.65$

Triclinic, $P \overline{1}$

$a=10.904(6) \AA$

$b=13.404(6) \AA$

$c=13.683(6) \AA$

$\alpha=88.97(4)^{\circ}$

$\beta=69.05(4)^{\circ}$

\section{Data collection}

Siemens R3m/V

diffractometer

Absorption correction: empirical (using intensity measurements)

Parkin et al. (1995)

$T_{\min }=0.85, T_{\max }=0.96$

6821 measured reflections

6447 independent reflections

\section{Refinement}

$R\left[F^{2}>2 \sigma\left(F^{2}\right)\right]=0.060$

$w R\left(F^{2}\right)=0.153$

$S=1.04$

6447 reflections

\section{Table 1}

Selected geometric parameters $\left(A,{ }^{\circ}\right)$

$\begin{array}{ll}\mathrm{N} 21-\mathrm{C} 1 & 1.366(4) \\ \mathrm{N} 21-\mathrm{C} 4 & 1.375(4) \\ \mathrm{N} 22-\mathrm{C} 9 & 1.373(4) \\ \mathrm{N} 22-\mathrm{C} 6 & 1.381(4) \\ \mathrm{N} 23-\mathrm{C} 14 & 1.373(4) \\ \mathrm{N} 23-\mathrm{C} 11 & 1.375(4) \\ \mathrm{N} 24-\mathrm{C} 16 & 1.372(4) \\ \mathrm{N} 24-\mathrm{C} 19 & 1.382(4) \\ \mathrm{C} 1-\mathrm{C} 20 & 1.417(4) \\ \mathrm{C} 1-\mathrm{C} 2 & 1.459(4)\end{array}$

$\gamma=77.88(4)^{\mathrm{o}}$

$V=1822.5(15) \AA^{3}$

$Z=2$

Mo $K \alpha$

$\mu=0.23 \mathrm{~mm}^{-1}$

$T=126(2) \mathrm{K}$

$0.7 \times 0.2 \times 0.2 \mathrm{~mm}$

4988 reflections with $I>2 \sigma(I)$

$R_{\text {int }}=0.036$

2 standard reflections

every 198 reflections

intensity decay: $<1 \%$

487 parameters

$\mathrm{H}$-atom parameters constrained

$\Delta \rho_{\max }=0.60 \mathrm{e} \AA^{-3}$

$\Delta \rho_{\min }=-0.80 \mathrm{e} \AA^{-3}$

$\begin{array}{ll}\mathrm{C} 7-\mathrm{C} 8 & 1.355(5) \\ \mathrm{C} 8-\mathrm{C} 9 & 1.426(4) \\ \mathrm{C} 9-\mathrm{C} 10 & 1.411(4) \\ \mathrm{C} 10-\mathrm{C} 11 & 1.400(4) \\ \mathrm{C} 11-\mathrm{C} 12 & 1.457(4) \\ \mathrm{C} 12-\mathrm{C} 13 & 1.345(4) \\ \mathrm{C} 13-\mathrm{C} 14 & 1.451(4) \\ \mathrm{C} 14-\mathrm{C} 15 & 1.405(4) \\ \mathrm{C} 15-\mathrm{C} 16 & 1.394(4) \\ \mathrm{C} 16-\mathrm{C} 17 & 1.428(4)\end{array}$


CIF access

$\begin{array}{llll}\mathrm{C} 2-\mathrm{C} 3 & 1.348(4) & \mathrm{C} 17-\mathrm{C} 18 & 1.357(4) \\ \mathrm{C} 2-\mathrm{N} 1 & 1.450(4) & \mathrm{C} 18-\mathrm{C} 19 & 1.433(4) \\ \mathrm{C} 3-\mathrm{C} 4 & 1.439(4) & \mathrm{C} 19-\mathrm{C} 20 & 1.399(4) \\ \mathrm{C} 4-\mathrm{C} 5 & 1.408(4) & \mathrm{N} 1-\mathrm{O} 1 & 1.226(3) \\ \mathrm{C} 5-\mathrm{C} 6 & 1.393(4) & & 1.241(3) \\ \mathrm{C} 6-\mathrm{C} 7 & 1.428(4) & \mathrm{C} 11-\mathrm{C} 10-\mathrm{C} 9 & \\ \mathrm{C} 1-\mathrm{N} 21-\mathrm{C} 4 & 106.6(2) & \mathrm{N} 23-\mathrm{C} 11-\mathrm{C} 10 & 124.4(3) \\ \mathrm{C} 9-\mathrm{N} 22-\mathrm{C} 6 & 110.3(2) & \mathrm{N} 23-\mathrm{C} 11-\mathrm{C} 12 & 126.0(3) \\ \mathrm{C} 14-\mathrm{N} 23-\mathrm{C} 11 & 105.0(2) & \mathrm{C} 10-\mathrm{C} 11-\mathrm{C} 12 & 110.6(3) \\ \mathrm{C} 16-\mathrm{N} 24-\mathrm{C} 19 & 110.6(2) & \mathrm{C} 13-\mathrm{C} 12-\mathrm{C} 11 & 123.4(3) \\ \mathrm{N} 21-\mathrm{C} 1-\mathrm{C} 20 & 125.9(3) & \mathrm{C} 12-\mathrm{C} 13-\mathrm{C} 14 & 106.7(3) \\ \mathrm{N} 21-\mathrm{C} 1-\mathrm{C} 2 & 108.5(2) & \mathrm{N} 23-\mathrm{C} 14-\mathrm{C} 15 & 106.6(3) \\ \mathrm{C} 20-\mathrm{C} 1-\mathrm{C} 2 & 125.6(3) & \mathrm{C} 15-\mathrm{C} 14-\mathrm{C} 13 & 126.1(3) \\ \mathrm{C} 3-\mathrm{C} 2-\mathrm{N} 13 & 111.0(3) \\ \mathrm{C} 3-\mathrm{C} 2-\mathrm{C} 1 & 121.2(3) & \mathrm{C} 16-\mathrm{C} 15-\mathrm{C} 14 & 122.8(3) \\ \mathrm{N} 1-\mathrm{C} 2-\mathrm{C} 1 & 108.4(3) & \mathrm{N} 24-\mathrm{C} 16-\mathrm{C} 15 & 125.7(3) \\ \mathrm{C} 2-\mathrm{C} 3-\mathrm{C} 4 & 130.0(2) & \mathrm{N} 24-\mathrm{C} 16-\mathrm{C} 17 & 126.7(3) \\ \mathrm{N} 21-\mathrm{C} 4-\mathrm{C} 5 & 105.7(3) & \mathrm{C} 15-\mathrm{C} 16-\mathrm{C} 17 & 106.5(2) \\ \mathrm{N} 21-\mathrm{C} 4-\mathrm{C} 3 & 126.3(3) & \mathrm{C} 18-\mathrm{C} 17-\mathrm{C} 16 & 126.9(3) \\ \mathrm{C} 5-\mathrm{C} 4-\mathrm{C} 3 & 110.8(2) & \mathrm{C} 17-\mathrm{C} 18-\mathrm{C} 19 & 108.5(3) \\ \mathrm{C} 6-\mathrm{C} 5-\mathrm{C} 4 & 122.9(3) & \mathrm{N} 24-\mathrm{C} 19-\mathrm{C} 20 & 108.4(3) \\ \mathrm{N} 22-\mathrm{C} 6-\mathrm{C} 5 & 125.8(3) & \mathrm{N} 24-\mathrm{C} 19-\mathrm{C} 18 & 126.6(3) \\ \mathrm{N} 22-\mathrm{C} 6-\mathrm{C} 7 & 126.6(3) & \mathrm{C} 20-\mathrm{C} 19-\mathrm{C} 18 & 106.0(2) \\ \mathrm{C} 5-\mathrm{C} 6-\mathrm{C} 7 & 106.0(3) & \mathrm{C} 19-\mathrm{C} 20-\mathrm{C} 1 & 127.3(3) \\ \mathrm{C} 8-\mathrm{C} 7-\mathrm{C} 6 & 127.4(3) & \mathrm{O} 1-\mathrm{N} 1-\mathrm{N} 1-\mathrm{C} 2 & 124.3(3) \\ \mathrm{C} 7-\mathrm{C} 8-\mathrm{C} 9 & 108.7(3) & 123.7(3) \\ \mathrm{N} 22-\mathrm{C} 9-\mathrm{C} 10 & 108.3(3) & 118.8(2) \\ \mathrm{N} 22-\mathrm{C} 9-\mathrm{C} 8 & 126.5(3) & 117.4(2) \\ \mathrm{C} 10-\mathrm{C} 9-\mathrm{C} 8 & 126.9(3) & & \end{array}$

\section{Acknowledgements}

This work was supported by grants from the Fonds der Chemischen Industrie and the Deutsche Forschungsgemeinschaft (Se543/2-4 and /3-1).

\section{References}

+mu1041+Dahal, S., Nethaji, M. \& Krishnan, V. (1994). Acta Cryst. C50, 314-317.

Giraudeau, A., Callot, H. J., Jordan, J., Ezhar, I. \& Gross, M. (1979). J. Am. Chem. Soc. 101, 3857-3862.

Hope, H. (1994). Prog. Inorg. Chem. 41, 1-13.

Jaquinod, L., Gros, C., Olmstead, M. M., Antolovich, M. \& Smith, K. M. (1996). Chem. Commun. 1475-1476.

Ozette, K., Leduc, P., Palacio, M., Bartoli, J.-F., Barkigia, K. M., Fajer, J., Battioni, P. \& Mansuy, D. (1997). J. Am. Chem. Soc. 119, 6442-, 6443.

Parkin, S. R., Moezzi, B. \& Hope, H. (1995). J. Appl. Cryst. 28, 53-56.

Sheldrick, G. M. (1990). Acta Cryst. A46, 467-473.

Sheldrick, G. M. (1993). SHELXL93. Program for the Refinement of Crystal Structures. University of Göttingen, Germany. 


\section{CIF access}

Sheldrick, G. M. (1994). SHEXLTL-Plus, Vers. 5.03, Program for Crystal Structure Solution and Refinement, Siemens Analytical Instruments Inc., Madison, WI, USA.

\section{Scheme 1}

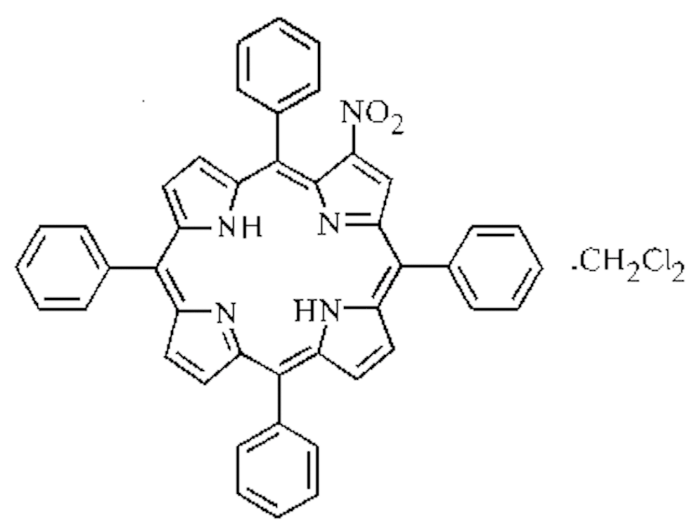




\section{supplementary materials}




\section{7-Nitro-5, 10,15,20-tetraphenylporphyrin}

\section{Crystal data}

$\mathrm{C}_{44} \mathrm{H}_{29} \mathrm{~N}_{5} \mathrm{O}_{2} \cdot \mathrm{C}_{1} \mathrm{H}_{2} \mathrm{Cl}_{2}$

$M_{r}=744.65$

Triclinic, $P \overline{\mathrm{I}}$

$a=10.904$ (6) $\AA$

$b=13.404(6) \AA$

$c=13.683$ (6) $\AA$

$\alpha=88.97(4)^{\circ}$

$\beta=69.05(4)^{\circ}$

$\gamma=77.88(4)^{\circ}$

$V=1822.5(15) \AA^{3}$

$Z=2$

Data collection

Siemens R3m/V

diffractometer

Radiation source: normal-focus sealed tube

Monochromator: graphite

$T=126(2) \mathrm{K}$

$\omega$-scans

Absorption correction: empirical (using intensity measurements)

Parkin et al. (1995)

$T_{\min }=0.85, T_{\max }=0.96$

6821 measured reflections

6447 independent reflections

4988 reflections with $I>2 \sigma(I)$

\section{Refinement}

Refinement on $F^{2}$

Least-squares matrix: full

$R\left[F^{2}>2 \sigma\left(F^{2}\right)\right]=0.060$

$w R\left(F^{2}\right)=0.153$

$S=1.04$

6447 reflections

487 parameters

Primary atom site location: structure-invariant direct methods
$F_{000}=772$

$D_{\mathrm{x}}=1.357 \mathrm{Mg} \mathrm{m}^{-3}$

Melting point: $\mathrm{n} / \mathrm{d} \mathrm{K}$

Mo $K \alpha$ radiation

$\lambda=0.71073 \AA$

Cell parameters from 35 reflections

$\theta=22-25^{\circ}$

$\mu=0.23 \mathrm{~mm}^{-1}$

$T=126(2) \mathrm{K}$

Parallelepiped, red

$0.7 \times 0.2 \times 0.2 \mathrm{~mm}$
$R_{\text {int }}=0.036$
$\theta_{\max }=25.1^{\circ}$
$\theta_{\min }=1.6^{\circ}$
$h=-11 \rightarrow 12$
$k=-15 \rightarrow 15$

$l=0 \rightarrow 16$

2 standard reflections

every 198 reflections

intensity decay: $<1 \%$
Secondary atom site location: difference Fourier map

Hydrogen site location: calc

$\mathrm{H}$-atom parameters constrained

Calculated $w=1 /\left[\sigma^{2}\left(F_{\mathrm{o}}{ }^{2}\right)+(0.0649 P)^{2}+1.7996 P\right]$

where $P=\left(F_{\mathrm{o}}{ }^{2}+2 F_{\mathrm{c}}{ }^{2}\right) / 3$ ?

$\Delta \rho_{\max }=0.60$ e $\AA^{-3}$

$\Delta \rho_{\min }=-0.80$ e $\AA^{-3}$

Extinction correction: none 


\section{Special details}

Geometry. All e.s.d.'s (except the e.s.d. in the dihedral angle between two 1.s. planes) are estimated using the full covariance matrix. The cell e.s.d.'s are taken into account individually in the estimation of e.s.d.'s in distances, angles and torsion angles; correlations between e.s.d.'s in cell parameters are only used when they are defined by crystal symmetry. An approximate (isotropic) treatment of cell e.s.d.'s is used for estimating e.s.d.'s involving 1.s. planes.

Refinement. Hydrogen atoms were placed in calculated positions and refined using a riding model. The $\beta$ - and pyrrole hydrogen atoms were initially located in difference maps. An absorption correction was performed using the program XABS2 (Parkin, Moezzi \& Hope, 1995). All calculations were performed using the SHELXTL-Plus program system (Sheldrick, 1994).

Fractional atomic coordinates and isotropic or equivalent isotropic displacement parameters $\left(\AA^{2}\right)$

\begin{tabular}{|c|c|c|c|c|}
\hline & $x$ & $y$ & $z$ & $U_{\text {iso }} * / U_{\text {eq }}$ \\
\hline $\mathrm{N} 21$ & -0.3788 (2) & $0.6208(2)$ & $0.8117(2)$ & $0.0273(5)$ \\
\hline $\mathrm{N} 22$ & $-0.4756(2)$ & $0.7199(2)$ & $1.0248(2)$ & $0.0320(6)$ \\
\hline $\mathrm{H} 22$ & $-0.4956(2)$ & $0.7326(2)$ & $0.9683(2)$ & $0.038^{*}$ \\
\hline $\mathrm{N} 23$ & -0.6259 (2) & $0.8905(2)$ & $0.9534(2)$ & $0.0295(5)$ \\
\hline $\mathrm{N} 24$ & -0.5330 (2) & $0.7926(2)$ & $0.7418(2)$ & $0.0275(5)$ \\
\hline $\mathrm{H} 24$ & $-0.5232(2)$ & $0.7754(2)$ & $0.8014(2)$ & $0.033^{*}$ \\
\hline $\mathrm{C} 1$ & -0.3529 (3) & $0.5820(2)$ & $0.7130(2)$ & $0.0256(6)$ \\
\hline $\mathrm{C} 2$ & $-0.2817(3)$ & $0.4754(2)$ & $0.7026(2)$ & $0.0276(6)$ \\
\hline $\mathrm{C} 3$ & -0.2625 & $0.4522(2)$ & $0.7930(2)$ & $0.0304(6)$ \\
\hline H3 & $-0.2182(3)$ & $0.3886(2)$ & $0.8087(2)$ & $0.037 *$ \\
\hline $\mathrm{C} 4$ & -0.3230 & $0.5440(2)$ & $0.8609(2)$ & $0.0280(6)$ \\
\hline $\mathrm{C} 5$ & $-0.3196(3)$ & $0.5515(2)$ & $0.9623(2)$ & $0.0300(6)$ \\
\hline C51 & -0.2332 & $0.4645(2)$ & $0.9944(2)$ & $0.0286(6)$ \\
\hline C52 & -0.0952 (3) & $0.4392(2)$ & $0.9391(2)$ & $0.0360(7)$ \\
\hline H52 & $-0.0562(3)$ & $0.4766(2)$ & $0.8808(2)$ & $0.043^{*}$ \\
\hline $\mathrm{C} 53$ & -0.0135 & $0.3594(3)$ & $0.9687(3)$ & $0.0420(8)$ \\
\hline H53 & $0.0807(3)$ & $0.3425(3)$ & $0.9302(3)$ & $0.050 *$ \\
\hline $\mathrm{C} 54$ & -0.0690 & $0.3046(2)$ & $1.0537(3)$ & $0.0405(8)$ \\
\hline H54 & -0.0131 & $0.2507(2)$ & $1.0742(3)$ & $0.049 *$ \\
\hline C55 & -0.2059 (3) & $0.3287(3)$ & $1.1086(3)$ & $0.0413(8)$ \\
\hline H55 & -0.2443 & $0.2908(3)$ & $1.1666(3)$ & $0.050^{*}$ \\
\hline C56 & -0.2882 & $0.4081(2)$ & $1.0796(2)$ & $0.0366(7)$ \\
\hline H56 & -0.3824 & $0.4240(2)$ & $1.1180(2)$ & $0.044 *$ \\
\hline $\mathrm{C} 6$ & -0.3877 (3) & $0.6338(2)$ & $1.0357(2)$ & $0.0318(7)$ \\
\hline $\mathrm{C} 7$ & -0.3825 & $0.6460(2)$ & $1.1375(2)$ & $0.0370(7)$ \\
\hline $\mathrm{H} 7$ & $-0.3282(3)$ & $0.5997(2)$ & $1.1668(2)$ & $0.044^{*}$ \\
\hline $\mathrm{C} 8$ & $-0.4678(3)$ & $0.7346(3)$ & $1.1855(2)$ & $0.0401(8)$ \\
\hline H8 & -0.4849 (3) & $0.7603(3)$ & $1.2545(2)$ & $0.048^{*}$ \\
\hline C9 & $-0.5276(3)$ & $0.7829(2)$ & $1.1147(2)$ & $0.0319(7)$ \\
\hline $\mathrm{C} 10$ & $-0.6184(3)$ & $0.8785(2)$ & $1.1317(2)$ & $0.0316(7)$ \\
\hline C101 & -0.6745 (3) & $0.9271(2)$ & 1.2407 (2) & $0.0321(7)$ \\
\hline C102 & -0.7514 & $0.8766(3)$ & $1.3225(2)$ & $0.0406(8)$ \\
\hline H102 & $-0.7646(3)$ & $0.8113(3)$ & $1.3083(2)$ & $0.049^{*}$ \\
\hline C103 & -0.8084 (4) & $0.9205(3)$ & $1.4236(3)$ & $0.0469(9)$ \\
\hline
\end{tabular}




\begin{tabular}{|c|c|c|c|c|}
\hline H103 & -0.8612 (4) & $0.8856(3)$ & $1.4783(3)$ & $0.056^{*}$ \\
\hline C104 & -0.7889 & $1.0151(3)$ & $1.4456(3)$ & $0.0441(8)$ \\
\hline H104 & -0.8277 (3) & $1.0450(3)$ & $1.5152(3)$ & $0.053^{*}$ \\
\hline $\mathrm{C} 105$ & $-0.7123(3)$ & $1.0659(3)$ & $1.3654(3)$ & $0.0399(8)$ \\
\hline H105 & -0.6989 (3) & $1.1310(3)$ & $1.3801(3)$ & $0.048 *$ \\
\hline C106 & $-0.6554(3)$ & $1.0223(2)$ & $1.2638(2)$ & $0.0344(7)$ \\
\hline H106 & -0.6028 & $1.0576(2)$ & $1.2094(2)$ & $0.041^{*}$ \\
\hline $\mathrm{C} 11$ & -0.6608 & $0.9282(2)$ & $1.0547(2)$ & $0.0302(6)$ \\
\hline $\mathrm{C} 12$ & -0.7510 & $1.0286(2)$ & $1.0732(2)$ & $0.0367(7)$ \\
\hline H12 & $-0.7896(3)$ & $1.0700(2)$ & $1.1369(2)$ & $0.044 *$ \\
\hline $\mathrm{C} 13$ & -0.7692 & $1.0514(2)$ & $0.9825(2)$ & $0.0367(7)$ \\
\hline H13 & -0.8219 & $1.1125(2)$ & $0.9694(2)$ & $0.044 *$ \\
\hline $\mathrm{C} 14$ & $-0.6926(3)$ & $0.9646(2)$ & $0.9085(2)$ & $0.0303(6)$ \\
\hline $\mathrm{C} 15$ & -0.6893 (3) & $0.9604(2)$ & $0.8050(2)$ & $0.0292(6)$ \\
\hline C151 & -0.7725 & 1.0489 (2) & $0.7728(2)$ & $0.0289(6)$ \\
\hline C152 & $-0.9126(3)$ & $1.0675(2)$ & $0.8167(2)$ & $0.0340(7)$ \\
\hline H152 & -0.9548 (3) & $1.0235(2)$ & $0.8667(2)$ & $0.041^{*}$ \\
\hline C153 & -0.9909 (3) & $1.1501(3)$ & $0.7877(3)$ & $0.0416(8)$ \\
\hline H153 & $-1.0863(3)$ & $1.1623(3)$ & $0.8181(3)$ & $0.050 *$ \\
\hline $\mathrm{C} 154$ & -0.9302 & $1.2142(3)$ & $0.7150(3)$ & $0.0414(8)$ \\
\hline H154 & -0.9837 (3) & $1.2701(3)$ & $0.6948(3)$ & $0.050^{*}$ \\
\hline C155 & -0.7917 (3) & $1.1971(3)$ & $0.6716(3)$ & $0.0423(8)$ \\
\hline H155 & -0.7501 & $1.2418(3)$ & $0.6222(3)$ & $0.051^{*}$ \\
\hline $\mathrm{C} 156$ & -0.7129 & $1.1150(2)$ & $0.6997(2)$ & $0.0366(7)$ \\
\hline H156 & $-0.6176(3)$ & $1.1035(2)$ & $0.6690(2)$ & $0.044 *$ \\
\hline C16 & -0.6131 & $0.8816(2)$ & $0.7288(2)$ & $0.0290(6)$ \\
\hline $\mathrm{C} 17$ & -0.6017 (3) & $0.8780(2)$ & $0.6216(2)$ & $0.0316(7)$ \\
\hline H17 & -0.6479 (3) & $0.9285(2)$ & $0.5899(2)$ & $0.038^{*}$ \\
\hline $\mathrm{C} 18$ & -0.5137 (3) & $0.7905(2)$ & $0.5726(2)$ & $0.0310(7)$ \\
\hline H18 & -0.4858 & $0.7703(2)$ & $0.5004(2)$ & $0.037 *$ \\
\hline C19 & -0.4699 (3) & $0.7337(2)$ & $0.6484(2)$ & $0.0279(6)$ \\
\hline $\mathrm{C} 20$ & -0.3839 & $0.6372(2)$ & $0.6324(2)$ & $0.0266(6)$ \\
\hline C201 & -0.3151 & $0.5930(2)$ & $0.5222(2)$ & $0.0255(6)$ \\
\hline $\mathrm{C} 202$ & -0.3890 & $0.5730(2)$ & $0.4621(2)$ & $0.0294(6)$ \\
\hline H202 & -0.4845 & $0.5903(2)$ & $0.4907(2)$ & $0.035^{*}$ \\
\hline C203 & -0.3237 (3) & $0.5285(2)$ & $0.3617(2)$ & $0.0347(7)$ \\
\hline $\mathrm{H} 203$ & -0.3743 & $0.5139(2)$ & $0.3220(2)$ & $0.042 *$ \\
\hline C204 & -0.1835 & $0.5047(2)$ & $0.3184(2)$ & $0.0364(7)$ \\
\hline H204 & -0.1386 (3) & $0.4729(2)$ & $0.2498(2)$ & $0.044 *$ \\
\hline $\mathrm{C} 205$ & -0.1100 & $0.5276(2)$ & $0.3757(2)$ & $0.0334(7)$ \\
\hline H205 & $-0.0146(3)$ & $0.5137(2)$ & $0.3452(2)$ & $0.040^{*}$ \\
\hline C206 & -0.1748 & $0.5707(2)$ & $0.4772(2)$ & $0.0279(6)$ \\
\hline H206 & -0.1235 & $0.5852(2)$ & $0.5164(2)$ & $0.033^{*}$ \\
\hline N1 & $-0.2484(3)$ & $0.3972(2)$ & $0.6198(2)$ & $0.0334(6)$ \\
\hline $\mathrm{O} 1$ & $-0.3360(2)$ & $0.3861(2)$ & $0.5871(2)$ & $0.0425(6)$ \\
\hline $\mathrm{O} 2$ & $-0.1330(2)$ & $0.3427(2)$ & $0.5888(2)$ & $0.0426(6)$ \\
\hline C1S & -0.0197 (4) & $0.2456(3)$ & $0.3472(3)$ & $0.0638(12)$ \\
\hline H1S1 & -0.0005 & $0.2236(3)$ & $0.4107(3)$ & $0.077^{*}$ \\
\hline $\mathrm{H} 1 \mathrm{~S} 2$ & $-0.0986(4)$ & $0.3037(3)$ & $0.3689(3)$ & $0.077^{*}$ \\
\hline
\end{tabular}




$\begin{array}{lllll}\text { C11S } & -0.05989(11) & 0.14540(9) & 0.29498(7) & 0.0651(3) \\ \text { C12S } & 0.11905(9) & 0.28716(7) & 0.25975(8) & 0.0531(3)\end{array}$

Atomic displacement parameters $\left(\AA^{2}\right)$

\begin{tabular}{|c|c|c|c|c|c|c|}
\hline & $U^{11}$ & $U^{22}$ & $U^{33}$ & $U^{12}$ & $U^{13}$ & $U^{23}$ \\
\hline N21 & $0.0246(12)$ & $0.0304(13)$ & $0.0267(12)$ & $-0.0041(10)$ & $-0.0101(10)$ & $0.0032(10)$ \\
\hline N22 & $0.0352(14)$ & $0.0340(14)$ & $0.0259(13)$ & $0.0012(11)$ & $-0.0150(11)$ & $0.0014(11)$ \\
\hline $\mathrm{N} 23$ & 0.0307 (13) & $0.0291(13)$ & $0.0272(12)$ & $-0.0010(10)$ & $-0.0117(10)$ & $0.0016(10)$ \\
\hline $\mathrm{N} 24$ & 0.0299 (13) & $0.0282(13)$ & $0.0245(12)$ & $-0.0022(10)$ & $-0.0125(10)$ & $0.0025(10)$ \\
\hline $\mathrm{C} 1$ & $0.0223(14)$ & $0.0272(15)$ & $0.0278(14)$ & $-0.0055(12)$ & $-0.0098(12)$ & $0.0029(12)$ \\
\hline $\mathrm{C} 2$ & $0.0262(15)$ & $0.0270(15)$ & $0.0293(15)$ & $-0.0046(12)$ & $-0.0105(12)$ & $-0.0007(12)$ \\
\hline $\mathrm{C} 3$ & $0.030(2)$ & $0.028(2)$ & $0.034(2)$ & $-0.0053(12)$ & $-0.0133(13)$ & $0.0053(12)$ \\
\hline $\mathrm{C} 4$ & $0.0259(15)$ & 0.029 (2) & $0.0295(15)$ & $-0.0054(12)$ & -0.0109 (12) & $0.0034(12)$ \\
\hline $\mathrm{C} 5$ & $0.028(2)$ & $0.032(2)$ & $0.030(2)$ & $-0.0053(13)$ & $-0.0116(12)$ & $0.0057(12)$ \\
\hline C51 & $0.031(2)$ & 0.030 & $0.0278(15)$ & $-0.0032(12)$ & $-0.0148(12)$ & $0.0017(12)$ \\
\hline C52 & $0.030(2)$ & $0.043(2)$ & $0.034(2)$ & $-0.0066(14)$ & -0.0105 (13) & $0.0067(14)$ \\
\hline $\mathrm{C} 53$ & $0.028(2)$ & $0.046(2)$ & $0.046(2)$ & $0.0051(14)$ & $-0.0127(14)$ & $-0.002(2)$ \\
\hline C54 & $0.045(2)$ & 0.033 & 0.044 & $0.0041(15)$ & $-0.024(2)$ & $0.0040(14)$ \\
\hline C55 & $0.045(2)$ & 0.039 & $0.043(2)$ & $-0.010(2)$ & -0.019 (2) & $0.0151(15)$ \\
\hline C56 & $0.033(2)$ & $0.039(2)$ & $0.039(2)$ & $-0.0067(14)$ & $-0.0149(14)$ & $0.0089(14)$ \\
\hline C6 & $0.030(2)$ & 0.035 & $0.029(2)$ & $-0.0003(13)$ & $-0.0128(13)$ & $0.0019(13)$ \\
\hline C7 & $0.041(2)$ & $0.039(2)$ & $0.032(2)$ & $0.0019(14)$ & $-0.0186(14)$ & $0.0034(14)$ \\
\hline $\mathrm{C} 8$ & $0.045(2)$ & 0.045 & $0.030(2)$ & $0.000(2)$ & -0.0175 & -0.0013 \\
\hline C9 & $0.034(2)$ & $0.034(2)$ & $0.0264(15)$ & $-0.0018(13)$ & $-0.0138(13)$ & $0.0014(12)$ \\
\hline $\mathrm{C} 10$ & $0.032(2)$ & $0.032(2)$ & $0.031(2)$ & $-0.0053(13)$ & $-0.0125(13)$ & $0.0016(13)$ \\
\hline C101 & $0.029(2)$ & $0.038(2)$ & $0.028(2)$ & $-0.0017(13)$ & $-0.0114(13)$ & $0.0004(13)$ \\
\hline C102 & $0.050(2)$ & $0.036(2)$ & $0.034(2)$ & -0.008 (2) & $-0.0128(15)$ & $0.0014(14)$ \\
\hline C103 & 0.059 (2) & $0.050(2)$ & 0.028 & $-0.013(2)$ & $-0.012(2)$ & $0.0078(15)$ \\
\hline C104 & $0.043(2)$ & $0.056(2)$ & $0.030(2)$ & $-0.001(2)$ & $-0.0142(15)$ & $-0.004(2)$ \\
\hline $\mathrm{C} 105$ & $0.038(2)$ & $0.041(2)$ & $0.044(2)$ & $-0.0073(15)$ & $-0.0189(15)$ & $-0.0061(15)$ \\
\hline C106 & $0.030(2)$ & 0.041 & $0.032(2)$ & $-0.0078(14)$ & -0.0114 & $0.0032(13)$ \\
\hline C11 & $0.0273(15)$ & $0.032(2)$ & $0.0280(15)$ & $-0.0005(12)$ & $-0.0093(12)$ & $-0.0008(12)$ \\
\hline $\mathrm{C} 12$ & $0.036(2)$ & $0.036(2)$ & $0.033(2)$ & $0.0050(14)$ & $-0.0129(14)$ & $-0.0055(13)$ \\
\hline $\mathrm{C} 13$ & $0.037(2)$ & $0.032(2)$ & $0.036(2)$ & $0.0074(14)$ & -0.0151 & $-0.0043(13)$ \\
\hline $\mathrm{C} 14$ & $0.0271(15)$ & $0.031(2)$ & $0.032(2)$ & $-0.0014(12)$ & $-0.0116(12)$ & $0.0022(12)$ \\
\hline $\mathrm{C} 15$ & $0.0270(15)$ & $0.029(2)$ & $0.0301(15)$ & $-0.0031(12)$ & $-0.0105(12)$ & $0.0027(12)$ \\
\hline C151 & $0.033(2)$ & $0.0261(15)$ & $0.0286(15)$ & $-0.0011(12)$ & $-0.0150(13)$ & $-0.0003(12)$ \\
\hline C152 & $0.033(2)$ & 0.033 & 0.038 & $-0.0059(13)$ & $-0.0156(14)$ & $0.0051(13)$ \\
\hline C153 & $0.031(2)$ & $0.041(2)$ & $0.052(2)$ & $-0.0005(14)$ & -0.018 (2) & $0.002(2)$ \\
\hline C154 & $0.046(2)$ & $0.034(2)$ & $0.045(2)$ & $0.0027(15)$ & $-0.024(2)$ & $0.0038(15)$ \\
\hline $\mathrm{C} 155$ & $0.047(2)$ & $0.036(2)$ & 0.044 & -0.005 (2) & $-0.018(2)$ & $0.0130(15)$ \\
\hline C156 & $0.030(2)$ & $0.036(2)$ & $0.041(2)$ & $-0.0033(13)$ & $-0.0122(14)$ & $0.0059(14)$ \\
\hline $\mathrm{C} 16$ & $0.0279(15)$ & $0.030(2)$ & $0.0301(15)$ & $-0.0043(12)$ & $-0.0136(12)$ & $0.0053(12)$ \\
\hline $\mathrm{C} 17$ & $0.032(2)$ & $0.032(2)$ & $0.031(2)$ & $-0.0012(13)$ & -0.0153 & $0.0045(13)$ \\
\hline $\mathrm{C} 18$ & $0.035(2)$ & $0.034(2)$ & $0.0263(15)$ & $-0.0036(13)$ & $-0.0154(13)$ & $0.0039(12)$ \\
\hline C19 & $0.0280(15)$ & $0.028(2)$ & $0.0298(15)$ & $-0.0059(12)$ & $-0.0129(12)$ & $0.0030(12)$ \\
\hline $\mathrm{C} 20$ & $0.0261(14)$ & 0.028 & $0.0279(14)$ & $-0.0085(12)$ & $-0.0115(12)$ & $0.0028(12)$ \\
\hline
\end{tabular}




\section{supplementary materials}

$\begin{array}{lllllll}\mathrm{C} 201 & 0.0293(15) & 0.0229(14) & 0.0253(14) & -0.0044(12) & -0.0120(12) & 0.0057(11) \\ \mathrm{C} 202 & 0.031(2) & 0.028(2) & 0.032(2) & -0.0069(12) & -0.0148(13) & 0.0055(12) \\ \text { C203 } & 0.043(2) & 0.037(2) & 0.031(2) & -0.0123(14) & -0.0202(14) & 0.0050(13) \\ \text { C204 } & 0.043(2) & 0.036(2) & 0.027(2) & -0.0098(14) & -0.0091(14) & -0.0008(13) \\ \text { C205 } & 0.028(2) & 0.033(2) & 0.035(2) & -0.0045(13) & -0.0068(13) & 0.0022(13) \\ \text { C206 } & 0.0282(15) & 0.0259(15) & 0.032(2) & -0.0055(12) & -0.0146(12) & 0.0059(12) \\ \text { N1 } & 0.0390(15) & 0.0289(14) & 0.0380(14) & -0.0050(12) & -0.0223(12) & 0.0071(11) \\ \text { O1 } & 0.0496(14) & 0.0383(13) & 0.0526(14) & -0.0119(11) & -0.0329(12) & 0.0051(10) \\ \text { O2 } & 0.0422(13) & 0.0352(13) & 0.0467(13) & 0.0077(10) & -0.0204(11) & -0.0059(10) \\ \text { C1S } & 0.067(3) & 0.055(2) & 0.053(2) & -0.025(2) & 0.004(2) & -0.009(2) \\ \text { C11S } & 0.0702(7) & 0.0852(8) & 0.0459(5) & -0.0450(6) & -0.0119(5) & -0.0019(5) \\ \text { C12S } & 0.0466(5) & 0.0408(5) & 0.0662(6) & -0.0150(4) & -0.0104(4) & 0.0024(4)\end{array}$

Geometric parameters $\left(\AA,{ }^{\circ}\right)$

$\begin{array}{ll}\mathrm{N} 21-\mathrm{C} 1 & 1.366(4) \\ \mathrm{N} 21-\mathrm{C} 4 & 1.375(4) \\ \mathrm{N} 22-\mathrm{C} 9 & 1.373(4) \\ \mathrm{N} 22-\mathrm{C} 6 & 1.381(4) \\ \mathrm{N} 23-\mathrm{C} 14 & 1.373(4) \\ \mathrm{N} 23-\mathrm{C} 11 & 1.375(4) \\ \mathrm{N} 24-\mathrm{C} 16 & 1.372(4) \\ \mathrm{N} 24-\mathrm{C} 19 & 1.382(4) \\ \mathrm{C} 1-\mathrm{C} 20 & 1.417(4) \\ \mathrm{C} 1-\mathrm{C} 2 & 1.459(4) \\ \mathrm{C} 2-\mathrm{C} 3 & 1.348(4) \\ \mathrm{C} 2-\mathrm{N} 1 & 1.450(4) \\ \mathrm{C} 3-\mathrm{C} 4 & 1.439(4) \\ \mathrm{C} 4-\mathrm{C} 5 & 1.408(4) \\ \mathrm{C} 5-\mathrm{C} 6 & 1.393(4) \\ \mathrm{C} 5-\mathrm{C} 51 & 1.503(4) \\ \mathrm{C} 51-\mathrm{C} 52 & 1.390(4) \\ \mathrm{C} 51-\mathrm{C} 56 & 1.396(4) \\ \mathrm{C} 52-\mathrm{C} 53 & 1.394(4) \\ \mathrm{C} 53-\mathrm{C} 54 & 1.382(5) \\ \mathrm{C} 54-\mathrm{C} 55 & 1.379(5) \\ \mathrm{C} 55-\mathrm{C} 56 & 1.390(4) \\ \mathrm{C} 6-\mathrm{C} 7 & 1.428(4) \\ \mathrm{C} 7-\mathrm{C} 8 & 1.355(5) \\ \mathrm{C} 8-\mathrm{C} 9 & 1.426(4) \\ \mathrm{C} 9-\mathrm{C} 10 & 1.411(4) \\ \mathrm{C} 10-\mathrm{C} 11 & 1.400(4) \\ \mathrm{C} 10-\mathrm{C} 101 & 1.495(4) \\ \mathrm{C} 101-\mathrm{C} 106 & 1.395(4) \\ \mathrm{C} 101-\mathrm{C} 102 & 1.398(4) \\ \mathrm{C} 102-\mathrm{C} 103 & 1.381(5) \\ \mathrm{C} 1-\mathrm{N} 21-\mathrm{C} 4 & 106.6(2) \\ \mathrm{C} 9-\mathrm{N} 22-\mathrm{C} 6 & 110.3(2) \\ \mathrm{C} 14-\mathrm{N} 23-\mathrm{C} 11 & 105.0(2) \\ & \\ & \end{array}$

$\begin{array}{ll}\mathrm{C} 103-\mathrm{C} 104 & 1.385(5) \\ \mathrm{C} 104-\mathrm{C} 105 & 1.387(5) \\ \mathrm{C} 105-\mathrm{C} 106 & 1.385(4) \\ \mathrm{C} 11-\mathrm{C} 12 & 1.457(4) \\ \mathrm{C} 12-\mathrm{C} 13 & 1.345(4) \\ \mathrm{C} 13-\mathrm{C} 14 & 1.451(4) \\ \mathrm{C} 14-\mathrm{C} 15 & 1.405(4) \\ \mathrm{C} 15-\mathrm{C} 16 & 1.394(4) \\ \mathrm{C} 15-\mathrm{C} 151 & 1.498(4) \\ \mathrm{C} 151-\mathrm{C} 152 & 1.394(4) \\ \mathrm{C} 151-\mathrm{C} 156 & 1.397(4) \\ \mathrm{C} 152-\mathrm{C} 153 & 1.394(4) \\ \mathrm{C} 153-\mathrm{C} 154 & 1.381(5) \\ \mathrm{C} 154-\mathrm{C} 155 & 1.380(5) \\ \mathrm{C} 155-\mathrm{C} 156 & 1.386(4) \\ \mathrm{C} 16-\mathrm{C} 17 & 1.428(4) \\ \mathrm{C} 17-\mathrm{C} 18 & 1.357(4) \\ \mathrm{C} 18-\mathrm{C} 19 & 1.433(4) \\ \mathrm{C} 19-\mathrm{C} 20 & 1.399(4) \\ \mathrm{C} 20-\mathrm{C} 201 & 1.492(4) \\ \mathrm{C} 201-\mathrm{C} 206 & 1.397(4) \\ \mathrm{C} 201-\mathrm{C} 202 & 1.404(4) \\ \mathrm{C} 202-\mathrm{C} 203 & 1.379(4) \\ \mathrm{C} 203-\mathrm{C} 204 & 1.394(5) \\ \mathrm{C} 204-\mathrm{C} 205 & 1.382(4) \\ \mathrm{C} 205-\mathrm{C} 206 & 1.385(4) \\ \mathrm{N} 1-\mathrm{O} 1 & 1.226(3) \\ \mathrm{N} 1-\mathrm{O} 2 & 1.241(3) \\ \mathrm{C} 15-\mathrm{C} 11 \mathrm{~S} & 1.735(4) \\ \mathrm{C} 1 \mathrm{~S}-\mathrm{C} 12 \mathrm{~S} & 1.751(4) \\ \mathrm{C} 105-\mathrm{C} 106-\mathrm{C} 101 & \\ \mathrm{~N} 23-\mathrm{C} 11-\mathrm{C} 10 & 120.6(3) \\ \mathrm{N} 23-\mathrm{C} 11-\mathrm{C} 12 & 126.0(3) \\ & 110.6(3) \\ & \end{array}$




\section{supplementary materials}

\begin{tabular}{|c|c|c|c|}
\hline $\mathrm{C} 16-\mathrm{N} 24-\mathrm{C} 19$ & $110.6(2)$ & $\mathrm{C} 10-\mathrm{C} 11-\mathrm{C} 12$ & $123.4(3)$ \\
\hline $\mathrm{N} 21-\mathrm{C} 1-\mathrm{C} 20$ & $125.9(3)$ & $\mathrm{C} 13-\mathrm{C} 12-\mathrm{C} 11$ & $106.7(3)$ \\
\hline $\mathrm{N} 21-\mathrm{C} 1-\mathrm{C} 2$ & $108.5(2)$ & $\mathrm{C} 12-\mathrm{C} 13-\mathrm{C} 14$ & $106.6(3)$ \\
\hline $\mathrm{C} 20-\mathrm{C} 1-\mathrm{C} 2$ & $125.6(3)$ & $\mathrm{N} 23-\mathrm{C} 14-\mathrm{C} 15$ & $126.1(3)$ \\
\hline $\mathrm{C} 3-\mathrm{C} 2-\mathrm{N} 1$ & $121.2(3)$ & $\mathrm{N} 23-\mathrm{C} 14-\mathrm{C} 13$ & $111.0(3)$ \\
\hline $\mathrm{C} 3-\mathrm{C} 2-\mathrm{C} 1$ & $108.4(3)$ & $\mathrm{C} 15-\mathrm{C} 14-\mathrm{C} 13$ & $122.8(3)$ \\
\hline $\mathrm{N} 1-\mathrm{C} 2-\mathrm{C} 1$ & $130.0(2)$ & $\mathrm{C} 16-\mathrm{C} 15-\mathrm{C} 14$ & $125.7(3)$ \\
\hline $\mathrm{C} 2-\mathrm{C} 3-\mathrm{C} 4$ & $105.7(3)$ & $\mathrm{C} 16-\mathrm{C} 15-\mathrm{C} 151$ & $116.9(2)$ \\
\hline $\mathrm{N} 21-\mathrm{C} 4-\mathrm{C} 5$ & $126.3(3)$ & $\mathrm{C} 14-\mathrm{C} 15-\mathrm{C} 151$ & $117.4(2)$ \\
\hline $\mathrm{N} 21-\mathrm{C} 4-\mathrm{C} 3$ & $110.8(2)$ & $\mathrm{C} 152-\mathrm{C} 151-\mathrm{C} 156$ & $118.6(3)$ \\
\hline $\mathrm{C} 5-\mathrm{C} 4-\mathrm{C} 3$ & $122.9(3)$ & $\mathrm{C} 152-\mathrm{C} 151-\mathrm{C} 15$ & $120.0(3)$ \\
\hline $\mathrm{C} 6-\mathrm{C} 5-\mathrm{C} 4$ & $125.8(3)$ & $\mathrm{C} 156-\mathrm{C} 151-\mathrm{C} 15$ & $121.4(3)$ \\
\hline $\mathrm{C} 6-\mathrm{C} 5-\mathrm{C} 51$ & $116.5(3)$ & $\mathrm{C} 153-\mathrm{C} 152-\mathrm{C} 151$ & $120.5(3)$ \\
\hline $\mathrm{C} 4-\mathrm{C} 5-\mathrm{C} 51$ & $117.7(3)$ & $\mathrm{C} 154-\mathrm{C} 153-\mathrm{C} 152$ & $120.1(3)$ \\
\hline $\mathrm{C} 52-\mathrm{C} 51-\mathrm{C} 56$ & $118.7(3)$ & $\mathrm{C} 155-\mathrm{C} 154-\mathrm{C} 153$ & $119.9(3)$ \\
\hline $\mathrm{C} 52-\mathrm{C} 51-\mathrm{C} 5$ & $119.9(3)$ & $\mathrm{C} 154-\mathrm{C} 155-\mathrm{C} 156$ & $120.4(3)$ \\
\hline $\mathrm{C} 56-\mathrm{C} 51-\mathrm{C} 5$ & $121.4(3)$ & $\mathrm{C} 155-\mathrm{C} 156-\mathrm{C} 151$ & $120.5(3)$ \\
\hline $\mathrm{C} 51-\mathrm{C} 52-\mathrm{C} 53$ & $120.5(3)$ & $\mathrm{N} 24-\mathrm{C} 16-\mathrm{C} 15$ & $126.7(3)$ \\
\hline $\mathrm{C} 54-\mathrm{C} 53-\mathrm{C} 52$ & $120.2(3)$ & $\mathrm{N} 24-\mathrm{C} 16-\mathrm{C} 17$ & $106.5(2)$ \\
\hline $\mathrm{C} 55-\mathrm{C} 54-\mathrm{C} 53$ & $119.6(3)$ & $\mathrm{C} 15-\mathrm{C} 16-\mathrm{C} 17$ & $126.9(3)$ \\
\hline $\mathrm{C} 54-\mathrm{C} 55-\mathrm{C} 56$ & $120.5(3)$ & $\mathrm{C} 18-\mathrm{C} 17-\mathrm{C} 16$ & $108.5(3)$ \\
\hline $\mathrm{C} 55-\mathrm{C} 56-\mathrm{C} 51$ & $120.4(3)$ & $\mathrm{C} 17-\mathrm{C} 18-\mathrm{C} 19$ & $108.4(3)$ \\
\hline $\mathrm{N} 22-\mathrm{C} 6-\mathrm{C} 5$ & $126.6(3)$ & $\mathrm{N} 24-\mathrm{C} 19-\mathrm{C} 20$ & $126.6(3)$ \\
\hline $\mathrm{N} 22-\mathrm{C} 6-\mathrm{C} 7$ & $106.0(3)$ & $\mathrm{N} 24-\mathrm{C} 19-\mathrm{C} 18$ & $106.0(2)$ \\
\hline $\mathrm{C} 5-\mathrm{C} 6-\mathrm{C} 7$ & $127.4(3)$ & $\mathrm{C} 20-\mathrm{C} 19-\mathrm{C} 18$ & $127.3(3)$ \\
\hline $\mathrm{C} 8-\mathrm{C} 7-\mathrm{C} 6$ & $108.7(3)$ & $\mathrm{C} 19-\mathrm{C} 20-\mathrm{C} 1$ & $124.3(3)$ \\
\hline $\mathrm{C} 7-\mathrm{C} 8-\mathrm{C} 9$ & $108.3(3)$ & $\mathrm{C} 19-\mathrm{C} 20-\mathrm{C} 201$ & $117.2(2)$ \\
\hline $\mathrm{N} 22-\mathrm{C} 9-\mathrm{C} 10$ & $126.5(3)$ & $\mathrm{C} 1-\mathrm{C} 20-\mathrm{C} 201$ & $118.5(2)$ \\
\hline $\mathrm{N} 22-\mathrm{C} 9-\mathrm{C} 8$ & $106.6(3)$ & $\mathrm{C} 206-\mathrm{C} 201-\mathrm{C} 202$ & $118.9(3)$ \\
\hline $\mathrm{C} 10-\mathrm{C} 9-\mathrm{C} 8$ & $126.9(3)$ & $\mathrm{C} 206-\mathrm{C} 201-\mathrm{C} 20$ & $120.0(2)$ \\
\hline $\mathrm{C} 11-\mathrm{C} 10-\mathrm{C} 9$ & $124.4(3)$ & $\mathrm{C} 202-\mathrm{C} 201-\mathrm{C} 20$ & $121.2(2)$ \\
\hline $\mathrm{C} 11-\mathrm{C} 10-\mathrm{C} 101$ & $118.9(3)$ & $\mathrm{C} 203-\mathrm{C} 202-\mathrm{C} 201$ & $120.4(3)$ \\
\hline $\mathrm{C} 9-\mathrm{C} 10-\mathrm{C} 101$ & $116.7(3)$ & $\mathrm{C} 202-\mathrm{C} 203-\mathrm{C} 204$ & $120.2(3)$ \\
\hline $\mathrm{C} 106-\mathrm{C} 101-\mathrm{C} 102$ & $118.4(3)$ & $\mathrm{C} 205-\mathrm{C} 204-\mathrm{C} 203$ & $119.8(3)$ \\
\hline $\mathrm{C} 106-\mathrm{C} 101-\mathrm{C} 10$ & $122.5(3)$ & $\mathrm{C} 204-\mathrm{C} 205-\mathrm{C} 206$ & $120.4(3)$ \\
\hline $\mathrm{C} 102-\mathrm{C} 101-\mathrm{C} 10$ & $119.1(3)$ & $\mathrm{C} 205-\mathrm{C} 206-\mathrm{C} 201$ & $120.3(3)$ \\
\hline $\mathrm{C} 103-\mathrm{C} 102-\mathrm{C} 101$ & $120.8(3)$ & $\mathrm{O} 1-\mathrm{N} 1-\mathrm{O} 2$ & $123.7(3)$ \\
\hline $\mathrm{C} 102-\mathrm{C} 103-\mathrm{C} 104$ & $120.2(3)$ & $\mathrm{O} 1-\mathrm{N} 1-\mathrm{C} 2$ & $118.8(2)$ \\
\hline $\mathrm{C} 103-\mathrm{C} 104-\mathrm{C} 105$ & $119.7(3)$ & $\mathrm{O} 2-\mathrm{N} 1-\mathrm{C} 2$ & $117.4(2)$ \\
\hline $\mathrm{C} 104-\mathrm{C} 105-\mathrm{C} 106$ & $120.2(3)$ & $\mathrm{C} 11 \mathrm{~S}-\mathrm{C} 1 \mathrm{~S}-\mathrm{C} 12 \mathrm{~S}$ & $113.5(2)$ \\
\hline
\end{tabular}

